\title{
Dilemma of the swollen optic disc: a fluorescein retinal angiography study
}

\author{
N. E. F. CARTLidge, R. C. Y. NG, AND P. J. B. TILlEY \\ From the Department of Neurology, Royal Victoria Infirmary, Newcastle upon Tyne NE1 4LP
}

SUMMARY In all of 100 consecutive patients referred for retinal angiography with a clinical diagnosis of probable or possible papilloedema a definite diagnosis was made from neurological assessment, neuroradiological investigations, and follow-up. The cases have been divided into 2 groupsthose with proved papilloedema and those which have been called either normal or pseudopapilloedema. In all cases the retinal angiograms showed normal or increased optic disc vascularity and normal or increased early and late disc fluorescence. On the basis of these alone clear differences could be shown between the groups. All cases with papilloedema showed a combination of either excess late fluorescence with excess early fluorescence or excess late fluorescence with excess disc vascularity. Only 2 cases of pseudopapilloedema (both with optic disc drusen) showed this combination, and in both the pattern of disc fluorescence was different from that seen in papilloedema. Retinal angiography therefore seems to be of considerable value in differentiating true from pseudopapilloedema, though this is possible only when each stage of the angiogram is carefully studied.

The difficulties in differentiating pseudopapilloedema from true papilloedema are well known to ophthalmologists and neurologists. They become more difficult when symptoms of intracranial disease are vague and a decision to undertake potentially dangerous neuroradiological investigations is entirely dependent upon a correct interpretation of the disc changes (Hoyt and Pont, 1962). While papilloedema may often be diagnosed by conventional ophthalmoscopy in certain cases there may be doubt about the interpretation of the changes in the optic disc. In such instances diagnosis may be facilitated by retinal angiography (Barrios, 1963; David et al., 1963; Miller et al., 1965; Blair and Walsh, 1969; Bynke and Aberg, 1970).

After intravenous injection of sodium fluorescein retinal angiograms are said to differentiate papilloedema from pseudopapilloedema both in the early and later stages after injection (Miller et al., 1965). Depending on the degree of disc swelling, angiograms done early after injection in patients with papilloedema show capillary dilatation, dye leakage, and microaneurysm formation. These changes are said to be absent in patients with pseudopapilloedema. In the later stages after injection there is leakage of dye beyond the margins of the disc (Barrios, 1963;

Address for reprints: Dr N. E. F. Cartlidge, Department of Neurology, Royal Victoria Infirmary, Newcastle upon Tyne NE1 4LP.
Blair and Walsh, 1969). A third feature, a tendency of the veins to retain fluorescence, is said to occur in papilloedema (David et al., 1963) and not in normal cases.

Initial optimism about the usefulness of this technique in differentiating true papilloedema from pseudopapilloedema, however, has not been entirely justified. Late fluorescence spreading beyond the margins of the disc is absent from some cases of papilloedema (Bynke and Aberg, 1970) and Sanders's classification of 5 types of papilloedema based on retinal angiograms emphasises some of the difficulties (Sanders, 1969).

Our own experience convinced us that a controlled study of this problem was needed. We have therefore reviewed the last 100 consecutive cases referred to us with a diagnosis of probable or possible papilloedema.

\section{Patients and methods}

The patients were referred for retinal angiography from a wide area around Newcastle. Most were from physicians within the Royal Victoria Infirmary, from the neurosurgeons and neurologists at the Regional Neurological Centre, or from the neurological and ophthalmological units at the Royal Victoria Infirmary. None were referred directly 
from general practitioners. All the patients referred by the various consultants were thought from the appearance of the optic disc to have either probable or possible papilloedema. Many had symptoms such as headache, dizziness, or visual failure which were thought possibly to indicate intracranial disease.

Colour photographs were taken of both discs, using a Zeiss Contax camera body loaded with Kodachrome II film (ASA 125, DIN 22), after pupillary dilatation with a combination of $1 \%$ cyclopentolate hydrochloride (Midrilate) and 10\% phenylephrine hydrochloride.

For fluorescein angiography the fundus camera was connected to a Zeiss-Siemens flash generator and a Robot Recorder camera. The setting was further modified (Rosen, 1967) by inserting a fluorescence excitation filter (Ilford Bright Spectrum Blue 622) over the No. 7 aperture of the illuminating diaphragm and a barrier filter (Kodak Wratten 15) placed in the masking cone in the camera body. The first photograph was taken as soon as the intravenous injection of $3 \mathrm{ml} 25 \%$ sodium fluorescein was completed, and about 5 to 8 seconds later the immediate phases of fluorescein flow were captured at 1-second intervals up to a period of 20 to 25 seconds after the injection. Later photographs were taken from both discs at 1, 2, 5, 10, 20, and 30 minutes after injection.

Follow-up data were obtained in all 100 cases, and in all a final definitive diagnosis was reached without reference to the retinal angiograms. Patients were allocated into 2 groups-firstly, those with papilloedema and, secondly, those whom we have called normal or who had pseudopapilloedema.

Most patients were included in the papilloedema group on the basis of proved raised intracranial pressure. They either had a tumour, found at operation, or had been diagnosed as suffering from benign intracranial hypertension, shown in most cases by ventriculography. A number of other patients in whom a local cause for the papilloedema was thought likely were included in this group. Most of them presented with visual failure (often unilateral) and were referred primarily because of doubts about the diagnosis. While many would question the use of the term papilloedema in this context, all these cases clearly had optic disc oedema for which a local cause was thought responsible.

In all the patients in the normal or pseudopapilloedema group either neurological investigations were negative or there was no change in the optic disc appearance during prolonged follow-up. Many had normal ventriculograms or airencephalograms and most of the others had a normal CSF pressure measured at lumbar puncture. The remainder have been seen at repeated follow-up visits for as long as 3 years, and in all the fundal appearances have remained unchanged. In none of these cases has there been evidence of either raised intracranial pressure or a local cause for the disc appearance.

Black-and-white prints $(6 \times 6 \mathrm{~cm})$ were produced in all 100 cases showing the various stages after injection, as outlined above. These were examined by each of us without knowledge of the patients' diagnosis and the appearances noted. Optic disc vascularity was assessed as either normal or excessive from the appearances of the films taken within the first 20 seconds after injection. Early fluorescence was assessed as either normal or excessive on the films taken within 2 minutes of injection, and late fluorescence was assessed as either normal or excessive on the films taken 5 minutes and more after injection. These assessments were largely subjective, though based on our experience of the normal gained in the past 3 years.

\section{Results}

Forty-eight cases were diagnosed as papilloedema and the remaining 52 were classed as pseudopapilloedema or normal. In 30 patients raised intracranial pressure was the cause of the papilloedema. Table 1 shows their final diagnosis. The papilloedema in the other 18 cases was due to a local cause. Table 2 shows the final diagnosis reached in each case. The 2 undiagnosed cases both had unilateral visual impairment with the appearance of a swollen optic disc. In both the carotid angiogram and airencephalogram were normal and vision improved in both as the optic disc oedema decreased during follow-up. Neither case was thought by the clinician in charge or an ophthalmologist to be examples of papillitis. They may have been examples of retinal vasculitis (Hayreh, 1972).

Although clinically it was usually possible to separate patients with raised intracranial pressure from those with a local cause for the papilloedema this was rarely possible from the optic disc appearances alone, and the retinal angiogram appearances

Table 1 Final diagnosis in 30 patients with papilloedema due to intracranial pressure

\begin{tabular}{lc}
\hline Diagnosis & \\
\hline Cerebral tumour & 18 \\
Benign intracranial hypertension & 8 \\
Subdural haematoma & 3 \\
Leukaemic meningitis & 1 \\
& Total \\
\hline
\end{tabular}


Table 2 Final diagnosis in 18 patients with papilloedema due to a local cause

\begin{tabular}{lc}
\hline Diagnosis & \\
\hline Papillitis & 6 \\
Hypertensive retinopathy & 5 \\
Ischaemic papillitis & 3 \\
Retinal vasculitis & 1 \\
Juxta papillary choroiditis & 1 \\
Undiagnosed & 2 \\
& Total \\
\hline
\end{tabular}

in these patients in respect of the 3 features that we have considered-optic disc vascularity and early and late disc fluorescence-were indistinguishable. For the purpose of this analysis we have considered the 2 groups of papilloedema together.

The final diagnosis in many cases in the pseudopapilloedema group was not clear, though in all we were confident that we had excluded either intracranial disease or a local progressive lesion as a cause of the disc appearance. Most cases fell into the category of the 'structurally full disc' and the remainder we shall consider in the Discussion.

Table 3 shows an analysis of the 3 features of the retinal angiograms that we have discussed above, comparing their incidence in the 2 groups of cases. Excess disc vascularity, though more common in patients with papilloedema, was nevertheless present in $25 \%$ of those who were subsequently shown to have pseudopapilloedema or normal discs. All but 5 cases of papilloedema showed excess disc vascularity and all 5 were examples of mild or early papilloedema. Early disc fluorescence was thought to be excessive in $63 \%$ of the papilloedema cases but in only 2 in the pseudopapilloedema group. Late excess disc fluorescence was present in all cases of papilloedema but in only 4 cases $(8 \%)$ of pseudopapilloedema.

Table 3 Comparison of retinal angiogram appearances in patients with and without papilloedema

\begin{tabular}{llll}
\hline & \multicolumn{3}{l}{ Retinal angiogram appearance } \\
\cline { 2 - 4 } Clinical group & $\begin{array}{l}\text { Excess disc } \\
\text { vascularity }\end{array}$ & \multicolumn{2}{l}{ Excess disc fluorescence } \\
\cline { 2 - 4 } & $43(87 \%)$ & $30(63 \%)$ & Larly \\
\hline $\begin{array}{l}\text { Papilloedema } \\
(n=48)\end{array}$ & $13(100 \%)$ \\
$\begin{array}{l}\text { Pseudopapilloedema } \\
\text { or normal } \\
(n=52)\end{array}$ & $13(25 \%)$ & $12(4 \%)$ & $4(8 \%)$ \\
\hline
\end{tabular}

\section{Discussion}

The aim of this study was to try to define the usefulness of retinal angiography in differentiating true from pseudopapilloedema. In all of the 100 cases a final diagnosis was made independently of the retinal angiographic changes, and hence it was possible to analyse the retinal angiograms blind in an attempt objectively to spot differences between the 2 groups of cases. A high percentage of cases of papilloedema showed excess disc vascularity and excessive early and late disc fluorescence. All showed late disc fluorescence and only 5 did not show excess disc vascularity. All these late cases, however, showed early and late excess fluorescence of the disc. In other words all cases of papilloedema showed either a combination of excess disc vascularity and excess late fluorescence or a combination of early and late excess disc fluorescence (Table 3).

The retinal angiogram in papilloedema is best considered in relationship to the pathology of papilloedema. From whatever cause, papilloedema is a result of capillary dilatation on the optic disc with transudation of fluid into the prelaminar portion (Schutta and Hedges, 1971). The retinal angiogram demonstrates not only the excess vascularity but, since the fluorescein dye leaks from the normal vessels in a similar fashion to the oedema fluid, staining of the optic disc by the dye measures the amount of transudate. The characteristic angiographic changes of papilloedema seen in our cases can thus be explained (Barrios, 1963; David et al., 1963; Miller et al., 1965; Shikano and Shimizu, 1968; Blaire and Walsh, 1969; Rosen, 1969; Bynke and Aberg, 1970).

The proportion of cases with papilloedema which show such changes will depend not only on the quality of film but also on the severity of the papilloedema and rapidity of its onset. This is covered fully by Sanders (1969). In very early papilloedema (a stage before Sanders's early group) we might expect oedema (measured by excess disc fluorescence) to be present without excess vascularity. Two of our cases of papilloedema showed this. The other 3 cases of papilloedema with disc fluorescence but no increase in disc vascularity were mild cases in which the early films were of poor quality. Disc fluorescence is, as we have stated, probably our best objective measure of continued oedema fluid leakage into the disc, and was seen in all our cases of papilloedema except the very chronic onesSanders's vintage group (Sanders, 1969). Certainly all our cases of papilloedema showed excess late disc fluorescence, though only two-thirds showed early leakage. Early leakage, however, seems to be more a function of rapidity of development than of 
severity of papilloedema. Those of our cases without early excess disc fluorescence were examples of chronic papilloedema. In summary, therefore, retinal angiography is a sensitive and accurate method of detecting papilloedema.

Of those patients in our normal or pseudopapilloedema group $25 \%$ showed excess disc vascularity. In isolation, therefore, this sign seems to be of little value and emphasises the wide normal variation in optic disc vascularity. Excess disc fluorescence, however, was seen in only 5 cases and only 1 of these had both early and late disc fluorescence (Table 4). Case 25 showed excess vascularity and early and late fluorescence, case 65 excess vascularity and early fluorescence, cases 2 and 41 late fluorescence only, and case 69 excess vascularity and late fluorescence.

As already stated, all our cases of papilloedema showed either excess vascularity and late fluorescence or early fluorescence and late fluorescence. Cases 65, 2 , and 4 can be differentiated from true papilloedema on this basis alone and they will not be considered further. Cases 26 and 69, however, cannot be differentiated from papilloedema on the basis of pointers we have considered so far.

Case 26 was a 26-year-old woman who presented with headaches and was found to have abnormal fundi but no other abnormal neurological findings. Subsequent neurological investigation showed no abnormality. CSF pressure at lumbar puncture was normal. No change in the fundal appearance was seen during follow-up, though the patient's headaches improved. Clinically there was nothing to suggest that this patient had raised intracranial pressure or a local cause producing papilloedema, though retinal angiography showed excess vascularity and early and late fluorescence. Careful study of the angiograms, however, showed changes that were very different from those in a case of papilloedema (Fig. 1). In papilloedema of this degree the early leakage occurs in a very specific manner with the dye appearing as minute spots (micro-

Table 4 Excess disc vascularity and excess early and late disc fluorescence in 5 cases of pseudopapilloedema

\begin{tabular}{llll}
\hline & \multirow{2}{*}{$\begin{array}{l}\text { Excess disc } \\
\text { vascularity }\end{array}$} & \multicolumn{2}{l}{ Excess fluorescence } \\
\cline { 3 - 4 } Case No. & Present & Present & Late \\
\hline 26 & Present & Present & Absent \\
65 & Absent & Absent & Present \\
2 & Absent & Absent & Present \\
4 & Present & Absent & Present \\
69 & & &
\end{tabular}

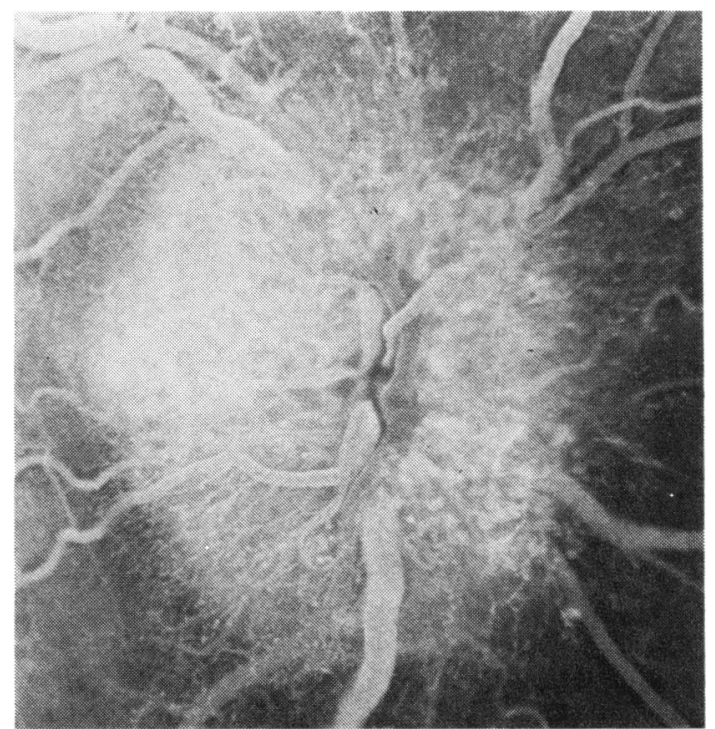

Fig. 1 Retinal angiogram taken 15 seconds after injection of fuorescein in patient with papilloedema due to benign intracranial hypertension (case 78)

aneurysms) on the disc surface coalescing and producing diffuse disc staining after 2 to 5 minutes. The early films of case 26 (Fig. 2) do not show any 'spotty leakage' and fluorescence of the disc occurred diffusely within 20 seconds of injection. This appearance is not seen in papilloedema so soon after injection. We believe that this case represents an example of buried drusen of the optic disc.

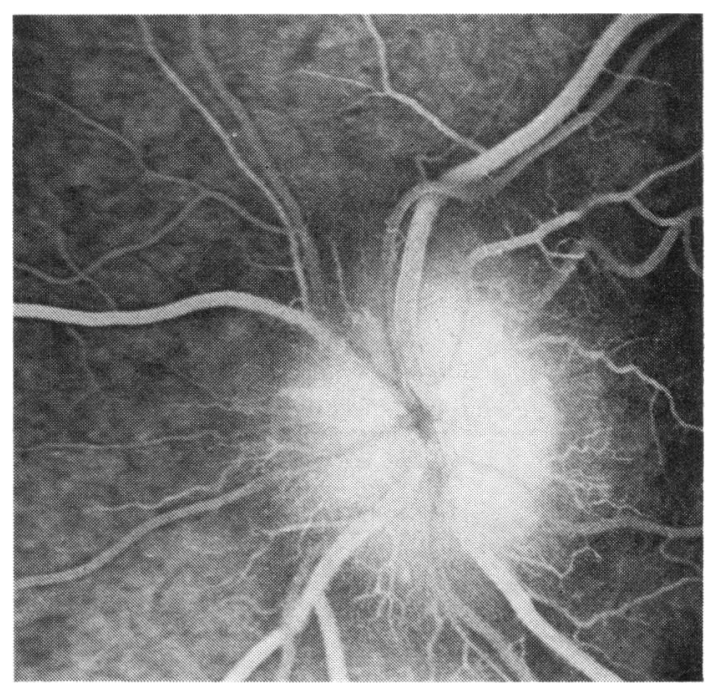

Fig. 2 Retinal angiogram taken 15 seconds after injection of fluorescein in case 26 
Case 69 was a 32-year-old man who presented with headaches. Investigation elsewhere had shown normal carotid angiogram and normal CSF pressure at lumbar puncture. Two years after the initial presentation the fundal appearance was unchanged and the headaches had gone. The retinal angiogram in this case showed excess vascularity of the disc but no early disc fluorescence (Fig. 3). The later stages, however, showed disc fluorescence spreading beyond the disc margin. The important diagnostic feature is seen in the early film (Fig. 3) where, at position $B$ the dye can be seen to be taken up in a local area. This focal early fluorescence is characteristic of giant drusen of the optic disc and vascularity of the disc is increased in these cases (Rosen 1969).

Our study shows that the retinal angiography is useful in differentiating true from pseudopapilloedema, though on the basis of excess disc vascu-

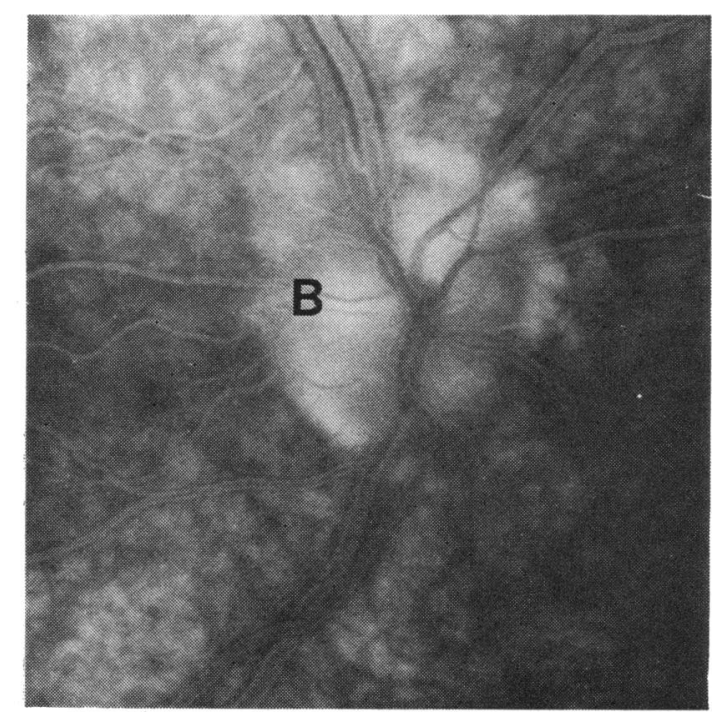

Fig. 3 Retinal angiogram taken 1 minute after injection of fluorescein in case 69. Note dye taken up in local area $(\mathrm{B})$ larity and excessive disc fluorescence it is not diagnostic. The combination of late disc fluorescence with either early fluorescence or excessive disc vascularity probably indicates papilloedema, but in view of the 2 exceptions among our cases we would emphasise the importance of meticulous attention to detail when examining retinal angiograms.

We gratefully acknowledge financial assistance from the Multiple Sclerosis Society of Great Britain and Northern Ireland.

\section{References}

Barrios, R. R. (1963). Fluorescein angioretinography as a diagnostic method for papilloedema. Acta Neurologica Latinoamerica, 9, 213-221.

Blair, C. J., and Walsh, F. B. (1969). Papilloedema, optic neuritis, and pseudopapilloedema. Transactions of the American Academy of Ophthalmology and Otolaryngology, 73, 914-920.

Bynke, H. B., and Aberg, L. (1970). Differentiation of papilloedema from pseudopapilloedema by fluorescein ophthalmoscopy. Acta Ophthalmologica, 48, 752-759.

David, N. J., Heyman, A., and Hart (1963). Fluorescence photography of retinal vessels and optic discs in papilloedema. Transactions of the American Neurological Association, 88, 155-156.

Hayreh, S. S. (1972). Benign retinal vasculitis. British Journal of Ophthalmology, 56, 652-656.

Hoyt, W. F., and Pont, M. E. (1962). Pseudopapilloedema, anomalous elevation of optic disc. Pitfalls in diagnosis and management. Journal of the American Medical Association. 181, 191-196.

Miller, S. J. H., Sanders, M. D., and Ffytche, T. J. (1965). Fluorescein fundus photography in the detection of early papilloedema and its differentiation from pseudopapilloedema. Lancet, 2, 651-654.

Rosen, E. S. (1967). Fluorescein (technical aspects). Manchester Medical Gazette, 47, 8-12.

Rosen, E. S. (1969). Fluorescence Photography of the Eye. Butterworths: London.

Sanders, M. D. (1969). A classification of papilloedema based on a fluorescein angiographic study. Transactions of the Ophthalmological Society, 89, 177-190.

Schutta, H. S., and Hedges, T. R. (1971). The pathogenesis of papilloedema. Journal of the Neurological Sciences, 12, 1-6.

Shikano, S., and Shimizu, K. (1968). Atlas of Fluorescence. Fundus Photography. Saunders: Philadelphia. 